\title{
Asymptotic behaviour of low density solutions to the generalized Becker-Döring equations *
}

\author{
Fernando Pestana DA COSTA \\ Instituto Superior Técnico, Departamento de Matemática \\ Avenida Rovisco Pais 1, P-1096 Lisboa, Portugal \\ e-mail: fernando.costa@math.ist.utl.pt
}

\begin{abstract}
The asymptotic behaviour of solutions to the generalized Becker-Döring equations is studied. It is proved that solutions converge strongly to a unique equilibrium if the initial density is sufficiently small.
\end{abstract}

\section{Introduction}

The mathematical theory of the coagulation-fragmentation equations has attracted considerable interested in recent years [1]-[7]. These equations are a model for the kinetics of cluster growth: denoting by $c_{j}=c_{j}(t) \geq 0$ the concentration of a cluster of $j$ identical particles at time $t \geq 0,(j=1,2,3, \ldots)$, and assuming the only reactions among clusters are elementary biparticle coagulation and binary monoparticle fragmentation, one gets the following kinetic equations, the coagulation-fragmentation equations,

$$
\dot{c}_{j}(t)=\frac{1}{2} \sum_{k=1}^{j-1} W_{j-k, k}(c(t))-\sum_{k=1}^{\infty} W_{j, k}(c(t)), \quad j=1,2,3, \ldots
$$

where $W_{j, k}(c)=a_{j, k} c_{j} c_{k}-b_{j, k} c_{j+k}$, and $a_{j, k}, b_{j, k}$ are the coagulation and the fragmentation rate coefficients, respectively, which are nonnegative constants satisfying $a_{j, k}=a_{k, j}, b_{j, k}=b_{k, j}$, for all $j, k$. The first sum in the right-hand side of (1.1) is defined to be zero if $j=1$.

With the physical interpretation of the phase variables $c_{j}$ given above, the quantity $\rho_{c}(t):=\sum_{j=1}^{\infty} j c_{j}(t)$ is the density of the system at time $t$, and physical considerations sugest the study of (1.1) in the Banach space of finite density sequences

*This work was done with the financial support of Fundação Calouste Gulbenkian. 
$X:=\left\{c=\left(c_{j}\right):\|c\|:=\left\|\left(j c_{j}\right)\right\|_{\ell^{1}}<\infty\right\}$. In fact, the only physically important solutions are the nonnegative ones and this is reflected in the definition of solution to (1.1), that includes the restriction $c(t) \in X^{+}:=X \cap\left\{\left(c_{j}\right): c_{j} \geq 0\right\}$ for all $t$ in its domain of definition [2]. In $X^{+}$we have $\|c(t)\|=\rho_{c}(t)$. We usually drop the subscript $c$ in $\rho_{c}(\cdot)$.

Naturally, properties of solutions to (1.1) dependent of the assumptions on the rate coefficients $a_{j, k}$ and $b_{j, k}$. One of the best understood and more interesting cases is obtained when $a_{j, k}=b_{j, k}=0$ if $\min \{j, k\}>1$ : the Becker-Döring system. This system has been extensively studied $[1,3,6,7]$ and, under convenient assumptions on the nonzero rate coefficients, it has been proved that solutions exhibit an asymptotic behaviour when $t \rightarrow \infty$ that can be physically interpreted as a dynamic phase transition $[1,3,7]$ : there exists a critical density $\rho_{s} \in(0, \infty)$ such that solutions to the Becker-Döring system with initial density $\rho_{0} \leq \rho_{s}$ converge strongly in $X$ to a unique equilibrium with density $\rho_{0}, c^{\rho_{0}}$, as $t \rightarrow \infty$ and if $\rho_{0}>\rho_{s}$ solutions converge weak* in $X$ (but not strongly) to an equilibrium $c^{\rho_{s}}$ with density $\rho_{s}$. The interpretation of this result as a phase transition is based on the fact that the excess density $\rho_{0}-\rho_{s}$ is transfered to larger and larger clusters as $t$ increases, corresponding in the limit $t \rightarrow \infty$ to the "condensation" of an infinite (macroscopic) cluster with that density. Two key ingredients to prove this result are the finite time density conservation of solutions, i.e., $\rho(t)=\rho_{0}$ for all $t \geq 0$, and the existence of a Lyapunov function $V_{z_{s}}$ which is sequentially weak* continuous in $X$ [3].

This kind of asymptotic behaviour is expected to hold for the general coagulation-fragmentation equations (1.1) under convenient assumptions on the rate coefficients. Motivated by the Becker-Döring case, it is natural to impose that, for all $j$ and $k$,

$$
a_{j, k} \leq K_{a}\left(j^{\alpha}+k^{\alpha}\right)
$$

for some constants $K_{a}>0, \alpha \in[0,1)$, since this condition ensures coagulation does not lead to a breakdown of density conservation [2].

Concerning the fragmentation coefficients, Carr and da Costa [5] considered the following "weak fragmentation" assumption

$$
\exists K_{f}>0: \forall r>1, \sum_{j=1}^{h(r)} j b_{j, r-j} \leq K_{f} r
$$

where $h(r)=[(r+1) / 2]$ and $[x]$ denote the integer part of $x$. Assuming $a_{j, k}$ and $b_{j, k}$ satisfy (1.2), (1.3), and some other hypoteses mainly concerning relations between these two type of coefficients (such as a "detailed balance" condition stating the existence of a positive sequence $Q_{j}$ such that $\left.a_{j, k} Q_{j} Q_{k}=b_{j, k} Q_{j+k}\right)$ it was proved in [5] that (i) system (1.1) possesses a Lyapunov function, (ii) there exists a critical density $\rho_{s}$ such that for each $0 \leq \rho \leq \rho_{s}$ there exists a unique equilibrium $c^{\rho}$ of (1.1) with density $\rho$ and there is no equilibria with $\rho>\rho_{s}$, and (iii) solutions $c(t)$ 
to (1.1) with density $\rho_{0}$ converge in the weak* sense as $t \rightarrow \infty$ to an equilibrium $c^{\rho}$ with $\rho \in\left[0, \min \left\{\rho_{0}, \rho_{s}\right\}\right]$, (see Theorems 5.2, 5.3 and 6.4 of $[5]$ ).

These results are direct extensions of what happens in the Becker-Döring system (compare with Theorem 4.7, Proposition 4.1 and Theorem 5.5 of [3], respectively), and, thus, they are a strong sugestion that the same type of phase transition behaviour should occur for (1.1), i.e., the convergence of $c(t)$ to an equilibrium $c^{\rho}$ as $t \rightarrow \infty$ should hold also in the strong topology of $X$ if $\rho_{0} \leq \rho_{s}$.

With this generality the problem is still open. Some progress in this direction was done in [5] by considering special cases of (1.1) for which $a_{j, k}=b_{j, k}=0$ if and only if $\min \{j, k\}>N$, for some positive integer constant $N$. The case $N=1$ is the Becker-Döring system, and cases with $N>1$ were called in [5] the generalized Becker-Döring equations. These are more general than the BeckerDöring equations but still considerably simpler than the coagulation-fragmentation system (1.1). It was proved in [5, Theorem 6.5] that solutions to the generalized Becker-Döring equations do exhibit the phase transition behaviour refered to above provided the initial data is rapidly decreasing (for some physically interesting coefficients the condition on the initial data is $\sum_{j=1}^{\infty} e^{\lambda j^{\mu}} c_{j}(0)<\infty$, where $\lambda>0$ and $\mu \in(0,1)$ are constants, see [5, Remark 6.7]). This result is an extension of what was proved for the Becker-Döring system in [3, Theorem 5.6].

The decay restriction on the initial data was removed for the Becker-Döring system in [1], and it is expected that the same can be done for the generalized Becker-Döring equations. In this paper we prove that this is true if the initial density $\rho_{0}$ is sufficiently small, namely, we prove that, under convenient hypotheses, solutions to the generalized Becker-Döring equations with initial data $c_{0} \in X^{+}$ with density $\rho_{0}$, converge strongly in $X$ to the unique equilibrium $c^{\rho_{0}}$ with density $\rho_{0}$ if $\rho_{0}<\rho_{N}$ for a constant $\rho_{N} \in\left(0, \rho_{s}\right]$ satisfying $\rho_{N} \sim \mathcal{O}\left(N^{-1}\right)$ as $N \rightarrow \infty$.

The method used to prove this result is a modified version of the one introduced by Ball and Carr for the Becker-Döring [1] and is based on estimates on the decay rates of $x_{n}(t):=\sum_{j=n}^{\infty} j c_{j}(t)$. In order to prove that solutions converge strongly in $X$ to some equilibrium state we need to prove precompactness of the orbit of the associated generalized dynamical system, i.e., we must show that $x_{n}(t) \rightarrow 0$ as $n \rightarrow \infty$ uniformly in $t$. We prove that if $\rho_{0}<\rho_{N}$ there exists a sequence $\lambda_{n} \rightarrow 0$ as $n \rightarrow \infty$ such that $x_{n}(t) \leq \lambda_{n}$ for all $t>0$, provided $x_{n}(0) \leq \lambda_{n}$. The sequences $\left(\lambda_{n}\right)$ are defined in a way similar to what was done in [1] and the fact that for each initial condition $c_{0}$ with small enough density a sequence $\left(\lambda_{n}\right)$ can be found satisfying $x_{n}(0) \leq \lambda_{n}$ follows from arguments analogous to the ones in that paper.

It was not possible to extend the method in order to deal with solutions with density larger than $\rho_{N}$, so the result for general $\rho_{0} \in\left[0, \rho_{s}\right]$ (if true) seems to require a new approach. Similarly, the result cannot be extended to the general equation 
(1.1): the proofs require $N<\infty$ and (1.1) is obtained, formally, by making $N=\infty$ (note also that $\rho_{N} \rightarrow 0$ as $N \rightarrow \infty$ ).

Nevertheless, although the general phase transition problem remains open, the result in this paper shows that the assumption on the decay rate of the initial data for the generalized Becker-Döring equations made in [5] is not necessary to obtain strong convergence to equilibria, at least when the initial density is sufficiently small.

The paper makes frequent use of ideas and results from $[1,5]$, and is organized as follows:

In Section 2 we briefly state the assumptions and basic results from [5] that are needed afterwards.

Section 3 contains the statement and proof of the main result (Theorem 3.1).

\section{Preliminaries}

In this section we state the results on the asymptotic behaviour of solutions that are needed in Section 3. For results on existence, uniqueness, density conservation, and regularity we refer to $[2,5]$.

Consider the following hypotheses

(H1) $a_{j, k} \leq K_{a}\left(j^{\alpha}+k^{\alpha}\right)$ for some constants $K_{a}>0$ and $\alpha \in[0,1)$.

(H2) For some constants $K_{f}, \kappa_{f}>0$ and $0<\beta<1$ such that, for all $r>1$,
1. $\sum_{j=1}^{h(r)} j b_{j, r-j} \leq K_{f} r$
2. $\sum_{j=1}^{h(r)} b_{j, r-j} \leq \kappa_{f} r^{\beta}$

(H3) For all $k \geq 1, a_{1, k}, b_{1, k}>0$.

(H4) There exists a sequence $\left(Q_{j}\right)$ such that

1. $Q_{1}=1$ and $a_{j, k} Q_{j} Q_{k}=b_{j, k} Q_{j+k}$ for all $j, k \geq 1$.

2. There exists a constant $K_{Q}>0$ such that $\left|\log Q_{j}-\log Q_{k}\right| \leq K_{Q} \mid j-$ $k \mid$, for all $j$ and $k$.

3. For all $j$ and $k,-\log Q_{j+k} \leq-\log Q_{j}-\log Q_{k}$.

4. $0<\liminf _{j \rightarrow \infty} Q_{j}^{1 / j} \leq \limsup _{j \rightarrow \infty} Q_{j}^{1 / j}<+\infty$.

By an equilibrium we mean a time independent solution of (1.1). It follows from $(\mathbf{H} 4-1)$ that $\bar{c}=\left(Q_{j} z^{j}\right)$ are equilibria of (1.1) provided $\|\bar{c}\|=\sum_{j=1}^{\infty} j Q_{j} z^{j}<\infty$. 
Hypothesis (H4-4) implies this series has a finite and positive radius of convergence $z_{s}=\left(\limsup _{j \rightarrow \infty} Q_{j}^{1 / j}\right)^{-1}$. Hence, for each $0 \leq z<z_{s}$, there is one equilibrium solution of the above form. The densities of these equilibria are

$$
F(z)=\sum_{j=1}^{\infty} j Q_{j} z^{j}
$$

and the critical density of (1.1) is defined by $\rho_{s}:=\sup _{0 \leq z<z_{s}} F(z) \in(0, \infty]$. If $\rho_{s}<\infty$ then $\rho_{s}=F\left(z_{s}\right)$. It is clear that no equilibria of the type under consideration has density larger than $\rho_{s}$. The problem of existence of equilibria not of the form $\bar{c}=\left(Q_{j} z^{j}\right)$ is negatively settled as an easy consequence of the fact that (1.1) has a Lyapunov function that satisfies a certain evolution equation. This important result also sets in motion the study of the asymptotic behaviour of solutions. We now turn our attention to it: Let $c \in X^{+}$and define $V: X^{+} \rightarrow \mathbb{R}$ by

$$
V(c):=\sum_{j=1}^{\infty} c_{j}\left(\log \frac{c_{j}}{Q_{j}}-1\right),
$$

where the summand is defined to be zero when $c_{j}=0$. Then the following result holds true

Proposition 2.1 [5, Theorem 5.2]

Assume $(\mathbf{H 1})-(\mathbf{H} 4)$. Let $T \in(0,+\infty]$ and let c be a solution to $(1.1)$ on $[0, T)$ with initial data $c_{0} \neq 0$. Then, for all $t \in[0, T)$

$$
V(c(t))=V(c(0))-\int_{0}^{t} D(c(s)) d s
$$

where

$$
D(c)=\sum_{j, k=1}^{\infty} W_{j, k}(c)\left[\log \left(Q_{j+k} c_{j} c_{k}\right)-\log \left(Q_{j} Q_{k} b_{j+k}\right)\right] \geq 0
$$

From (2.2) and (2.3) it follows that $V$ is non-increasing along solutions, and if $c=\bar{c}$ is an equilibrium then $\int_{0}^{t} D(\bar{c}) d s=0$ for all $t$, which, by $(\mathbf{H} 4-1)$ implies that $\bar{c}$ is of the form considered above.

In order to prove convergence to equilibria of solutions to (1.1) we need to assume

(H5) $b_{j, k} \leq o(j) o(k)$ as $j, k \rightarrow \infty$,

and to substitute (H4-4) by the following stronger hypothesis 
(H4-4a) The sequence $\left(Q_{j}^{1 / j}\right)$ converges to a positive number as $j \rightarrow \infty$.

Since a strongly bounded set in $X$ is weak* compact, and since the assumptions imply the apriori estimate $\|c(t)\|=\|c(0)\|=\rho_{0}$ holds true [2], it is natural to study first the convergence to equilibria in the weak* topology of $X$. We need the Lyapunov function to be weak* continuous in $X$, which the function $V$ defined above is not. However, using the fact that solutions conserve density, the functional $V_{z_{s}}(c):=V(c)-\|c\| \log z_{s}$ is also a Lyapunov function for (1.1), satisfies the energy equation $(2.3)$, and has the required continuity properties [3, 5$]$. Using this Lyapunov function we can then prove the following

Proposition 2.2 [5, Theorem 6.4] Assume $(\mathbf{H 1})-(\mathbf{H 5})^{1}$. Let $c$ be a solution of $(1.1)$ on $[0, \infty)$ with initial data $c(0)=$ $c_{0},\left\|c_{0}\right\|=\rho_{0}$. Let $c^{\rho}$ be the equilibrium of (1.1) with $\left\|c^{\rho}\right\|=\rho$. Then $c(t)$ converges weak* in $X$ to $c^{\rho}$ as $t \rightarrow \infty$ for some $\rho \in\left[0, \min \left\{\rho_{0}, \rho_{s}\right\}\right]$.

As pointed out in the Introduction, the identification of the density $\rho$ of the limit point (i.e., the distinction between weak and strong convergence) has not been accomplished in full generality, and, namely, to the best of our knowledge, nothing is known for the general equations (1.1).

Results in this direction were obtained in [5] under the following restriction:

(H6) There exists a positive integer $N>1$ such that $a_{j, k}=b_{j, k}=0$ if and only if $\min \{j, k\}>N$.

The case $N=1$ transforms (1.1) into the Becker-Döring equations, for which the problem was completely solved in $[1,3]$.

Assuming (H6) system (1.1) becomes the following generalized Becker-Döring system

$$
\left\{\begin{array}{rlrl}
\dot{c}_{1} & =-\sum_{k=1}^{\infty} W_{1, k}(c) & \\
\dot{c}_{j} & =\frac{1}{2} \sum_{k=1}^{j-1} W_{j-k, k}(c)-\sum_{k=1}^{\infty} W_{j, k}(c), & & 2 \leq j \leq N \\
\dot{c}_{j} & =\frac{1}{2} \sum_{k=1}^{N-1} W_{j-k, k}(c)-\sum_{k=1}^{N} W_{j, k}(c), & & N+1 \leq j \leq 2 N \\
\dot{c}_{j} & =\sum_{k=1}^{N} W_{j-k, k}(c)-\sum_{k=1}^{N} W_{j, k}(c), & & j \geq 2 N+1 .
\end{array}\right.
$$

We then have

\footnotetext{
${ }^{1}$ With (H4-4) changed to $(\mathbf{H 4 - 4 a )}$.
} 
Proposition 2.3 [5, Theorem 6.6] In addition to the assumptions of Proposition 2.2 consider (H6). Suppose there exists a constant $M>0$ and a positive integer $k_{0}$ such that, for all $\ell=1, \ldots, N$ and all $i>k_{0}$,

$$
\begin{aligned}
& 0 \leq b_{i, \ell}-a_{i, \ell} Q_{\ell} z_{s}^{\ell} \leq M \\
& 0 \leq b_{i-\ell, \ell}-a_{i, \ell} Q_{\ell} z_{s}^{\ell}
\end{aligned}
$$

Let $\rho_{s}<\infty$ and suppose $c_{0} \in X^{+}$satisfies $\sum_{j=1}^{\infty} c_{0 j} \cdot\left(Q_{j} z_{s}^{j}\right)^{-1}<\infty$. Suppose also that $c$ is the only solution of $(2.4)$ on $[0, \infty)$ with initial data $c_{0}$. Then:

(i) If $0 \leq \rho_{0} \leq \rho_{s}$ then $c(t) \rightarrow c^{\rho_{0}}$ strongly in $X$ as $t \rightarrow \infty$ and $\lim _{t \rightarrow \infty} V(c(t))=$ $V\left(c^{\rho_{0}}\right)$.

(ii) If $\rho_{0}>\rho_{s}$ then $c(t) \stackrel{*}{\rightarrow} c^{\rho_{s}}$ as $t \rightarrow \infty$, but not strongly in $X$, and $\lim _{t \rightarrow \infty} V(c(t))$ $=V\left(c^{\rho_{s}}\right)+\left(\rho_{0}-\rho_{s}\right) \log z_{s}$.

In this paper we need to change some of the above assumptions into slightly stronger ones, namely, we substitute (H5) by

(H5 $a$ ) There exists constants $K_{u} \geq K_{\ell}>0$ and $0 \leq \gamma<1$ such that, for all $k=1, \ldots, N$ and all $j \geq 1$,

$$
K_{\ell} j^{\gamma} \leq b_{j, k} \leq K_{u} j^{\gamma} .
$$

Define $K_{B}:=K_{\ell} / K_{u}$

We need also to change $(\mathbf{H} 4-4 a)$ into the stronger hypothesis

$(\mathbf{H} 4-4 b)$ The sequence $\left(Q_{j+1} / Q_{j}\right)$ converge to a positive number as $j \rightarrow \infty$.

Note that if this holds then (H4-4a) also holds and

$$
z_{s}^{-1}=\lim _{j \rightarrow \infty} Q_{j}^{1 / j}=\lim _{j \rightarrow \infty}\left(Q_{j+1} / Q_{j}\right) .
$$

\section{Results}

The main result of the paper is the following

Theorem 3.1 Assume $(\mathbf{H 1})-(\mathbf{H 6})^{2}$. Suppose there exists an integer $r_{0}$ such that, for all $z \in\left[0, z_{s}\right)$, all $\ell=1, \ldots, N$, and all $i>r_{0}$,

$$
b_{i-\ell, \ell}-a_{i, \ell} Q_{\ell} z^{\ell} \geq 0 .
$$

\footnotetext{
${ }^{2}$ With $(\mathbf{H 4}-4)$ changed to $(\mathbf{H 4}-4 b)$ and $(\mathbf{H 5})$ to $(\mathbf{H 5} a)$.
} 
Let $c(\cdot)$ be the only solution of $(2.4)$ on $[0, \infty)$ with initial data $c_{0} \in X^{+}$, and $\rho_{0}=\left\|c_{0}\right\|$. Let $\rho_{N}$ be the unique positive solution of $F^{-1}(\rho)=z_{s} K_{B} N^{-1}$, where $F^{-1}$ is the inverse function of $F$ defined by $(2.1)$. Consider $\rho_{0} \in\left[0, \rho_{N}\right)$, then $c(t) \longrightarrow c^{\rho_{0}}$ strongly in $X$ as $t \rightarrow \infty$ and $\lim _{t \rightarrow \infty} V(c(t))=V\left(c^{\rho_{0}}\right)$.

To prove Theorem 3.1 we need a result which is a generalization of [1, Theorem 2] and is given in Theorem 3.2 below. First we fix some notation: for $z \in\left(0, z_{s}\right)$ define

$$
\begin{aligned}
\tilde{\mu}_{j, k} & :=1-\frac{k Q_{k} z^{k} a_{j, k}}{j b_{j-k, k}} \\
\mu_{j} & :=\left(\min _{1 \leq k \leq N} \tilde{\mu}_{j, k}\right)^{-1} \\
\beta_{j} & :=\max _{1 \leq k \leq N} b_{j-k, k} \\
\chi_{j} & :=\max _{1 \leq k \leq N} \frac{j Q_{j} z^{j}}{(j-k) Q_{j-k} z^{j-k}} \\
\sigma_{n} & :=\sum_{j=n}^{n+N-1} \beta_{j} \chi_{j} \\
\nu_{n} & :=\beta_{n}^{-1} \mu_{n} \sigma_{n}
\end{aligned}
$$

Theorem 3.2 Assume (H1)-(H6) ${ }^{3}$, and (3.1). Let $\left(\lambda_{j}\right)$ be a positive nonincreasing sequence such that, for all $r \geq r_{0}$,

$$
\lambda_{j}-\lambda_{j+1} \geq \nu_{j}\left(\lambda_{j-1}-\lambda_{j}\right) .
$$

Let $c$ be the unique solution of $(2.4)$ on $[0, \infty)$ with initial data $c(0)=c_{0} \in X^{+}$, $\rho_{0}=\left\|c_{0}\right\|<\rho_{s}$. Then

$$
H(t):=\max \left\{\sup _{n \geq r_{0}+1} \lambda_{n}^{-1} x_{n}(t), \lambda_{r_{0}}^{-1} \rho_{0}\right\}
$$

is nonincreasing on $[0, \infty)$, where $x_{n}(t):=\sum_{j=n}^{\infty} j c_{j}(t)$.

Proof: We start by considering the finite $m$-dimensional truncation of (2.4) obtained by making $a_{j, k}=b_{j, k}=0$ if $j+k>m$. Let

$$
H^{(m)}(t):=\max \left\{\sup _{r_{0}+1 \leq n \leq m} \lambda_{n}^{-1} x_{n}^{(m)}(t), \lambda_{r_{0}}^{-1} \rho_{0}^{(m)}\right\}
$$

\footnotetext{
${ }^{3}$ With (H4-4) changed to $(\mathbf{H 4}-4 a)$.
} 
where $x_{n}^{(m)}(t)=\sum_{j=n}^{m} j c_{j}^{(m)}(t), c^{(m)}(\cdot)$ is the solution of the truncated problem, and $\rho_{0}^{(m)}=\sum_{j=1}^{m} j c_{0 j}$. It suffices to prove that, if $H^{(m)}(0)<\infty$ and $T>0$, then $H^{(m)}(t) \leq H^{(m)}(0)+\varepsilon$ for all $\varepsilon>0$ and all $t \in[0, T]$. Suppose this is false. Since $H^{(m)}(\cdot)$ is continuous, there exist an $\varepsilon>0$ and a least $s \in[0, T]$ such that $H^{(m)}(s)=K_{\varepsilon}:=H^{(m)}(0)+\varepsilon$. Since $H^{(m)}(0) \geq \lambda_{r_{0}}^{-1} \rho_{0}^{(m)}$ we conclude that $H^{(m)}(s)>\lambda_{r_{0}}^{-1} \rho_{0}^{(m)}$, which implies that $g(s):=\sup _{r_{0}+1 \leq n \leq m} \lambda_{n}^{-1} x_{n}^{(m)}(s)=K_{\varepsilon}$. Thus, there exists a smallest $n$ satisfying $r_{0}+1 \leq n \leq m$ such that

$$
\begin{aligned}
\lambda_{n}^{-1} x_{n}^{(m)}(s) & =K_{\varepsilon} \\
\lambda_{n-1}^{-1} x_{n-1}^{(m)}(s) & <K_{\varepsilon} \\
\lambda_{n+1}^{-1} x_{n+1}^{(m)}(s) & \leq K_{\varepsilon}
\end{aligned}
$$

and

$$
\dot{x}_{n}^{(m)}(s) \geq 0
$$

Without loss of generality, let $r_{0} \geq 2 N$. Then, by the definition of $x_{n}^{(m)}$ we have

$$
\begin{aligned}
\dot{x}_{n}^{(m)}= & \sum_{k=1}^{N} \sum_{j=n-k}^{n-1}(j+k) W_{j, k}\left(c^{(m)}\right)+\sum_{k=1}^{N} \sum_{j=n}^{m-k} k W_{j, k}\left(c^{(m)}\right) \\
= & \sum_{k=1}^{N} \sum_{j=n-k}^{n-1}(j+k) W_{j, k}\left(c^{(m)}\right)-\sum_{k=1}^{N} \sum_{j=m-k+1}^{m} k b_{j-k, k} c_{j}^{(m)}+ \\
& +\sum_{k=1}^{N}\left[\sum_{j=n}^{n+k-1} k a_{j, k} c_{j}^{(m)} c_{k}^{(m)}+\sum_{j=n+k}^{m-k} k a_{j, k} c_{j}^{(m)} c_{k}^{(m)}-\right. \\
\leq & \sum_{k=1}^{N} \sum_{j=n-k}^{n-1}(j+k)\left(a_{j, k} c_{j}^{(m)} c_{k}^{(m)}-b_{j, k} c_{j+k}^{(m)}\right)+\sum_{k=1}^{N} \sum_{j=n}^{n+k-1} k b_{j, k} c_{j}^{(m)} c_{j}^{(m)} c_{k}^{(m)} \\
& +\sum_{k=1}^{N} k \sum_{j=n+k}^{m-k}\left(a_{j, k} c_{k}^{(m)}-b_{j-k, k}\right) c_{j}^{(m)} .
\end{aligned}
$$

By Proposition 2.2, $c_{j}(t) \underset{t \rightarrow \infty}{\longrightarrow} Q_{j} z(\rho)^{j}$ for some $\rho \leq \rho_{0}<\rho_{s}$. Also $c_{j}^{(m)} \rightarrow c_{j}$ as $m \rightarrow \infty$. Thus, since $z(\rho)<z\left(\rho_{s}\right)=z_{s}$, we can choose $T>0$ and $z<z_{s}$ such that, for all $k=1, \ldots, N$, and all $t \geq T, c_{k}^{(m)}(t)<Q_{k} z^{k}$, for all sufficiently large $m$. Using this information and (3.1) the last double sum in (3.13) can be bounded 
above by zero:

$$
\sum_{k=1}^{N} k \sum_{j=n+k}^{m-k}\left(a_{j, k} c_{k}^{(m)}-b_{j-k, k}\right) c_{j}^{(m)} \leq \sum_{k=1}^{N} k \sum_{j=n+k}^{m-k}\left(a_{j, k} Q_{k} z^{k}-b_{j-k, k}\right) c_{j}^{(m)} \leq 0 .
$$

Hence, we conclude that

$$
\dot{x}_{n}^{(m)} \leq \sum_{k=1}^{N}\left[\sum_{j=n}^{n+k-1} k a_{j, k} c_{j}^{(m)} Q_{k} z^{k}+\sum_{j=n-k}^{n-1}(j+k)\left(a_{j, k} c_{j}^{(m)} Q_{k} z^{k}-b_{j, k} c_{j+k}^{(m)}\right)\right] .
$$

By definition of $x_{n}^{(m)}$ we have $c_{j}^{(m)}=\left(x_{j}^{(m)}-x_{j+1}^{(m)}\right) / j$ and thus, after some rearrangements,

$$
\begin{aligned}
& \dot{x}_{n}^{(m)} \leq \\
& \leq \sum_{k=1}^{N} \sum_{j=n}^{n+k-1}\left[j a_{j-k, k} Q_{k} z^{k} \frac{x_{j-k}^{(m)}-x_{j-k+1}^{(m)}}{j-k}-\right. \\
& \left.\quad-\left(j b_{j-k, k}-k Q_{k} z^{k} a_{j, k}\right) \frac{x_{j}^{(m)}-x_{j+1}^{(m)}}{j}\right] \\
& =\sum_{k=1}^{N} \sum_{j=n}^{n+k-1} b_{j-k, k}\left[\frac{j Q_{j} z^{j}}{(j-k) Q_{j-k} z^{j-k}}\left(x_{j-k}^{(m)}-x_{j-k+1}^{(m)}\right)-\tilde{\mu}_{j, k}\left(x_{j}^{(m)}-x_{j+1}^{(m)}\right)\right]
\end{aligned}
$$

Observing that $\left(x_{j}^{(m)}\right)$ is nonincreasing with $j$ and that, by (3.1), $\tilde{\mu}_{j, k}>0$ we have

$$
\begin{aligned}
\dot{x}_{n}^{(m)} & \leq \sum_{k=1}^{N} \sum_{j=n}^{n+k-1} \beta_{j}\left[\chi_{j}\left(x_{j-k}^{(m)}-x_{j-k+1}^{(m)}\right)-\mu_{j}^{-1}\left(x_{j}^{(m)}-x_{j+1}^{(m)}\right)\right] \\
& =\sum_{j=n}^{n+N-1} \sum_{k=j-n+1}^{N} \beta_{j}\left[\chi_{j}\left(x_{j-k}^{(m)}-x_{j-k+1}^{(m)}\right)-\mu_{j}^{-1}\left(x_{j}^{(m)}-x_{j+1}^{(m)}\right)\right] \\
& =\sum_{j=n}^{n+N-1} \beta_{j}\left[\chi_{j}\left(x_{n-1}^{(m)}-x_{j-N+1}^{(m)}\right)-\mu_{j}^{-1}\left(x_{j}^{(m)}-x_{j+1}^{(m)}\right)(N-j+n)\right] \\
& \leq-\sum_{j=n}^{n+N-1} \beta_{j} \mu_{j}^{-1}\left(x_{j}^{(m)}-x_{j+1}^{(m)}\right)+\left(x_{n-1}^{(m)}-x_{n}^{(m)}\right) \sum_{j=1}^{n+N-1} \beta_{j} \chi_{j} \\
& \leq-\beta_{n} \mu_{n}^{-1}\left(x_{n}^{(m)}-x_{n+1}^{(m)}\right)+\sigma_{n}\left(x_{n-1}^{(m)}-x_{n}^{(m)}\right) .
\end{aligned}
$$

By (3.9)-(3.12) we have

$$
x_{n-1}^{(m)}(s)-x_{n}^{(m)}(s)=x_{n}^{(m)}(s)\left(\frac{x_{n-1}^{(m)}(s)}{x_{n}^{(m)}(s)}-1\right)<
$$




$$
<x_{n}^{(m)}(s)\left(\frac{\lambda_{n-1}}{\lambda_{n}}-1\right)=K_{\varepsilon}\left(\lambda_{n-1}-\lambda_{n}\right),
$$

and

$$
\begin{aligned}
x_{n}^{(m)}(s)-x_{n+1}^{(m)}(s) & =x_{n}^{(m)}(s)\left(1-\frac{x_{n+1}^{(m)}(s)}{x_{n}^{(m)}(s)}\right) \geq \\
& \geq x_{n}^{(m)}(s)\left(1-\frac{\lambda_{n+1}}{\lambda_{n}}\right)=K_{\varepsilon}\left(\lambda_{n}-\lambda_{n+1}\right) .
\end{aligned}
$$

Thus, using (3.8),

$$
0 \leq \dot{x}_{n}^{(m)}(s)<K_{\varepsilon}\left[\sigma_{n}\left(\lambda_{n-1}-\lambda_{n}\right)-\beta_{n} \mu_{n}^{-1}\left(\lambda_{n}-\lambda_{n+1}\right)\right] \leq 0
$$

and this contradiction proves that $H^{(m)}(t) \leq H^{(m)}(0)+\varepsilon$, for all $\varepsilon>0$, and $t \in[0, T]$. Since $H^{(m)}(0) \leq H(0)$ it follows that, for all $t \in[0, T], n \geq r_{0}+1$, and $m$ sufficiently large,

$$
\lambda_{n}^{-1} \sum_{j=n}^{m} j c_{j}^{(m)}(t) \leq H(0)+\varepsilon .
$$

Letting $m \rightarrow \infty$ and taking the supremum over $n \geq r_{0}+1$ gives the result.

Before starting the prove Theorem 3.1 we need the following estimate

Lemma 3.1 Assume $(\mathbf{H 4}-4 b),(\mathbf{H 5} a)$ and (3.1). Then, for all $\mathcal{K}>1$, there exists a $R_{0} \geq r_{0}$ such that, for all $r \geq R_{0}$,

$$
\nu_{r} \leq \mathcal{K} K_{B}^{-1} N \frac{z}{z_{s}} .
$$

Proof: From (3.7) we have

$$
\nu_{r}=\beta_{r}^{-1} \mu_{r} \sigma_{r}=\frac{\sum_{k=r}^{r+N-1}\left(\max _{1 \leq p \leq N} b_{k-p, p}\right) \max _{1 \leq p \leq N} \frac{k Q_{k} z^{k}}{(k-p) Q_{k-p} z^{k-p}}}{\left(\max _{1 \leq k \leq N} b_{r-k, k}\right) \cdot \min _{1 \leq k \leq N}\left(1-\frac{k Q_{k} z^{k} a_{r, k}}{r b_{r-k, k}}\right)} .
$$

Observing that

$$
\begin{aligned}
{\left[\min _{1 \leq k \leq N}\left(1-\frac{k Q_{k} z^{k} a_{r, k}}{r b_{r-k, k}}\right)\right]^{-1} } & =\max _{1 \leq k \leq N}\left(1-\frac{k Q_{k} z^{k} a_{r, k}}{r b_{r-k, k}}\right)^{-1} \\
& =\max _{1 \leq k \leq N} \frac{r b_{r-k, k}}{r b_{r-k, k}-k Q_{k} z^{k} a_{r, k}} \\
& \leq \frac{r \max _{1 \leq k \leq N} b_{r-k, k}}{\min _{1 \leq k \leq N}\left(r b_{r-k, k}-k Q_{k} z^{k} a_{r, k}\right)}
\end{aligned}
$$


the product of the first two terms in (3.14) satisfy

$$
\begin{aligned}
\beta_{r}^{-1} \mu_{r} & \leq \frac{r}{\min _{1 \leq k \leq N}\left(r b_{r-k, k}-k Q_{k} z^{k} a_{r, k}\right)} \\
& =\frac{r}{\min _{1 \leq k \leq N}\left[(r-k) b_{r-k, k}+k\left(b_{r-k, k}-Q_{k} z^{k} a_{r, k}\right)\right]} \\
& \leq \frac{r}{r-N}\left(\min _{1 \leq k \leq N} b_{r-k, k}\right)^{-1} .
\end{aligned}
$$

For $\sigma_{r}$ we have the following

$$
\begin{aligned}
\sigma_{r} & =\sum_{k=r}^{r+N-1}\left(\max _{1 \leq p \leq N} b_{k-p, p}\right)\left(\max _{1 \leq p \leq N} \frac{k Q_{k} z^{k}}{(k-p) Q_{k-p} z^{k-p}}\right) \\
& \leq \sum_{k=r}^{r+N-1} \frac{k}{k-N} Q_{k} z^{k} \frac{\max _{1 \leq p \leq N} b_{k-p, p}}{\min _{1 \leq p \leq N} Q_{k-p} z^{k-p}} \\
& \leq \frac{r}{r-N} \sum_{k=r}^{r+N-1}\left(\max _{1 \leq p \leq N} b_{k-p, p}\right) \max _{1 \leq p \leq N} \frac{Q_{k}}{Q_{k-p}} z^{p} \\
& \leq \frac{r N}{r-N} \max _{r \leq k \leq r+N-1}\left(\left(\max _{1 \leq p \leq N} b_{k-p, p}\right) \max _{1 \leq p \leq N} \frac{Q_{k}}{Q_{k-p}} z^{p}\right) \\
& \leq \frac{r N}{r-N}\left[\left(\max _{\substack{r \leq k \leq r+N-1 \\
1 \leq p \leq N}} b_{k-p, p}\right) \cdot\left(\max _{\substack{r \leq k \leq r+N-1 \\
1 \leq p \leq N}} \frac{Q_{k}}{Q_{k-p}} z^{p}\right)\right] .
\end{aligned}
$$

Hence

$$
\begin{aligned}
\nu_{r} \leq & N\left(\frac{r}{r-N}\right)^{2} \cdot\left(\max _{\substack{r \leq k \leq r+N-1 \\
1 \leq p \leq N}} b_{k-p, p}\right) . \\
& \cdot\left(\min _{1 \leq p \leq N} b_{r-p, p}\right)^{-1} \cdot\left(\max _{\substack{r \leq k \leq r+N-1 \\
1 \leq p \leq N}} \frac{Q_{k}}{Q_{k-p}} z^{p}\right)
\end{aligned}
$$

Using (H5 $a)$

$$
\left(\max _{\substack{r \leq k \leq r+N-1 \\ 1 \leq p \leq N}} b_{k-p, p}\right) \cdot\left(\min _{1 \leq p \leq N} b_{r-p, p}\right)^{-1} \leq K_{B}^{-1}\left(\frac{r+N-2}{r-N}\right)^{\gamma}
$$


and since $Q_{j+1} / Q_{j} \rightarrow z_{s}^{-1}$ as $j \rightarrow \infty$ we obtain

$$
\begin{aligned}
\limsup _{r \rightarrow \infty} \max _{\substack{r \leq k \leq r+N-1 \\
1 \leq p \leq N}} \frac{Q_{k}}{Q_{k-p}} z^{p} & \leq \limsup _{k \rightarrow \infty} \max _{1 \leq p \leq N} \frac{Q_{k}}{Q_{k-p}} z^{p} \\
& =\max _{1 \leq p \leq N}\left[z^{p} \limsup _{k \rightarrow \infty} \prod_{j=1}^{p} \frac{Q_{k-j+1}}{Q_{k-j}}\right] \\
& =\max _{1 \leq p \leq N}\left(z / z_{s}\right)^{p} \\
& =z / z_{s} .
\end{aligned}
$$

By (3.15)-(3.17) we have

$$
\limsup _{r \rightarrow \infty} \nu_{r} \leq N K_{B}^{-1} \frac{z}{z_{s}}
$$

which proves the Lemma.

We need to recall from [1] the following definition and lemmas: Let $\nu_{j}>0$ for all $j \geq r_{0}$, and define the set

$$
\mathcal{S}:=\left\{\left(\lambda_{j}\right): \lambda_{j} \geq \lambda_{j+1} \geq 0 \forall j, \text { and } \lambda_{j}-\lambda_{j+1} \geq \nu_{j}\left(\lambda_{j-1}-\lambda_{j}\right) \forall j \geq r_{0}\right\} .
$$

Then we have

Lemma 3.2 [1, Lemma 3] The set $\mathcal{S}$ is closed under addition, multiplication by a nonnegative constant, and taking of infima.

Lemma 3.3 [1, Lemma 4] Suppose there exists a positive sequnce $\left(\eta_{j}\right) \in \mathcal{S}$ such that $\eta_{j} \rightarrow 0$ as $j \rightarrow \infty$. Let $\left(\psi_{j}\right)$ be any nonnegative sequence converging to zero. Then there exists a strictly positive sequence $\left(\hat{\lambda}_{j}\right) \in \mathcal{S}$ such that $\hat{\lambda}_{j} \geq \psi_{j}$ for all $j$, and $\hat{\lambda}_{j} \underset{j \rightarrow \infty}{\longrightarrow} 0$.

Proof of Theorem 3.1: We prove that $c(t) \stackrel{*}{\rightarrow} c^{\rho}$ as $t \rightarrow \infty$ for some $\rho<\rho_{s}$ implies $c(t) \rightarrow c^{\rho}$ strongly in $X$, provided $\rho<\rho_{N}$ where $\rho_{N}$ is the only positive solution of $F^{-1}(\rho)=z_{s} K_{B} N^{-1}$. Suppose $c_{j}(t)<Q_{j} z^{j}$ for some $z<z_{s}$, all $t \geq$ 0 , and all $j=1, \ldots, N$. By the argument following Eq.(3.13) in the proof of Theorem 3.2, we can always choose a new time origin for which this holds.

Define

$$
\eta_{j}=\sum_{r=j}^{\infty} r \gamma_{r}
$$

where $\gamma_{r}=1$ for $r \in\left\{0, \ldots, r_{0}-1\right\}$ and $r \gamma_{r}=\nu_{r}(r-1) \gamma_{r-1}$ for $r \geq r_{0}$. We prove that $\eta_{j} \rightarrow 0$ as $j \rightarrow \infty$, and $\left(\eta_{j}\right) \in \mathcal{S}$ :

By Lemma 3.1

$$
r \gamma_{r}=\left(r_{0}-1\right) \prod_{k=r_{0}}^{r} \nu_{r} \leq\left(r_{0}-1\right)\left(\frac{z}{z_{s} \kappa}\right)^{r-r_{0}+1}
$$


where $\kappa=\mathcal{K} K_{B} N^{-1}$. For $z<z_{s} \kappa$, the series $\sum_{j}\left(z / z_{s} \kappa\right)^{j}$ converges, which proves that $\left(\gamma_{r}\right) \in X$ and thus $\eta_{j} \rightarrow 0$ as $j \rightarrow \infty$. Furthermore,

$$
\eta_{j}=\sum_{r=j}^{\infty} r \gamma_{r} \geq \sum_{r=j+1}^{\infty} r \gamma_{r}=\eta_{j+1}
$$

and for $j \geq r_{0}$

$$
\eta_{j}-\eta_{j+1}=j \gamma_{j}=\nu_{j}(j-1) \gamma_{j-1}=\nu_{j}\left(\eta_{j-1}-\eta_{j}\right) .
$$

Hence $\left(\eta_{j}\right) \in \mathcal{S}$.

Since $c(t) \in X$ we have $x_{n}(0) \rightarrow 0$ as $n \rightarrow \infty$, for all $t \geq 0$. In particular, defining $\psi_{n}=x_{n}(0)$, we have $\psi_{n} \geq 0$ for all $n$ and $\psi_{n} \rightarrow \infty$ as $n \rightarrow \infty$. Let $\hat{\lambda}$ be the corresponding sequence given by Lemma 3.3. By Theorem 3.2 we have, for all $n>r_{0}$,

$$
\begin{aligned}
\hat{\lambda}_{n}^{-1} x_{n}(t) & \leq \sup _{n \geq r_{0}+1} \hat{\lambda}_{n}^{-1} x_{n}(t) \leq H(t) \\
& \leq H(0)=\max \left\{\sup _{n \geq r_{0}+1} \hat{\lambda}_{n}^{-1} x_{n}(0), \hat{\lambda}_{r_{0}}^{-1} \rho_{0}\right\} \\
& \leq \max \left\{1, \hat{\lambda}_{r_{0}}^{-1} \rho_{0}\right\} .
\end{aligned}
$$

Hence, for all $t \geq 0$ and all $n>r_{0}$,

$$
\sum_{j=n}^{\infty} j c_{j}(t) \leq \hat{\lambda}_{n} \max \left\{1, \hat{\lambda}_{r_{0}}^{-1} \rho_{0}\right\},
$$

and hence $c(t) \rightarrow c^{\rho_{0}}$ strongly in $X$ as $t \rightarrow \infty$. The asymptotic behaviour of $V(c(t))$ as $t \rightarrow \infty$ follows by the continuity properties of the Lyapunov function [3].

Note 3.1 With $F(z)$ given by $(2.1)$ we have $F(z) / z \rightarrow 1$ as $z \rightarrow 0$. Since $\rho_{N}=$ $F\left(z_{s} K_{B} N^{-1}\right)$, it follows that $\rho_{N}=z_{s} K_{B} N^{-1}+o\left(N^{-1}\right)$ as $N \rightarrow \infty$.

Acknowledgements: I thank J. Carr for several discussions.

\section{References}

[1] J.M. BALL, J. CARR, Asymptotic behaviour of solutions to the BeckerDöring equations for arbitrary initial data, Proc. Royal Soc. Edinburgh Sect. A 108, 109-116 (1988)

[2] J.M. BALL, J. CARR, The discrete coagulation-fragmentation equations: existence, uniqueness, and density conservation, J. Statist. Phys. 61, 203-234 (1990) 
[3] J.M. BALL, J. CARR, O. PENROSE, The Becker-Döring cluster equations: basic properties and asymptotic behaviour of solutions, Commun. Math. Phys. 104, 657-692 (1986)

[4] J. CARR, Asymptotic behaviour of solutions to the coagulation-fragmentation equations. I. The strong fragmentation case', Proc. Royal Soc. Edinburgh Sect. A 121, 231-244 (1992)

[5] J. CARR, F.P. da COSTA, Asymptotic behavior of solutions to the coagulation-fragmentation equations. II. Weak fragmentation, J. Statist. Phys. 77, 89-123 (1994)

[6] O. PENROSE, Metastable states for the Becker-Döring cluster equations, Commun. Math. Phys. 124, 515-541 (1989)

[7] M. SLEMROD, Trend to equilibrium in the Becker-Döring cluster equations, Nonlinearity 2, 429-443 (1989)

Received July 19, 1996 